\title{
A Simple Model for Predicting Foam Spread Over Liquids
}

\author{
BROR PERSSON and MARTIN DAHLBERG \\ Swedish National Testing and Research Institute \\ Box 857, S-501 15 BORAS, Sweden
}

\begin{abstract}
An approximate theoretical model of foam spreading on a liquid surface has been formulated. The model is analogous to the spreading of oil slicks on water surfaces. Viscous friction is assumed to be the dominating mechanism in opposing the foam spread. The friction is described by a lumped friction constant. The spreading process is decoupled from the mass transport due to evaporation and drainage of water contained in the foam. This work constitutes the first step in an attempt to formulate a model describing foam spread on a burning surface.
\end{abstract}

Preliminary experiments have been carried out with foam spreading in a circular water basin without external heating. The friction coefficient is determined from experimental data. The model fits reasonably well with the experimental results for a wide range of volume flow rates and foam expansion numbers. Thus it can be concluded that the model correctly describes the basic phenomena of the foam spread.

KEYWORDS: foam flow, radiation, mathematical model

\section{NOTATIONS}

$\boldsymbol{h}$ foam thickness (m)

$\boldsymbol{k}_{f}$ friction coefficient $\left(\mathrm{Ns} / \mathrm{m}^{3}\right)$

$\boldsymbol{u} \quad$ velocity $(\mathrm{m} / \mathrm{s})$

$L$ foam layer extension (1D) (m)

$\dot{v} \quad$ volume flow $\left(\mathrm{m}^{2} / \mathrm{s}\right.$ alt $\left.\mathrm{m}^{3} / \mathrm{s}\right)$

$\dot{m} \quad \operatorname{mass}$ flux $\left(\mathrm{kg} / \mathrm{m}^{2}, \mathrm{~s}\right)$

$\dot{q}_{r} \quad$ radiative flux $\left(\mathrm{W} / \mathrm{m}^{2}\right)$

$\boldsymbol{x} \quad$ longitudinal co-ordinate $(\mathrm{m})$

$\boldsymbol{R}$ foam extension (rot symm) (m)

$y \quad$ transverse co-ordinate $(\mathrm{m})$

$S$ foam expansion ratio $\left(\rho_{l} / \rho\right)$

$\beta \quad$ friction parameter $(1 / \mathrm{s})$

$t \quad$ time (sec)

$\mu \quad$ dynamic viscosity $\left(\mathrm{Ns} / \mathrm{m}^{2}\right)$

$\rho$ density of foam $\left(\mathrm{kg} / \mathrm{m}^{3}\right)$

$\tau_{f}$ friction force $\left(\mathrm{N} / \mathrm{m}^{2}\right)$

Subscripts:

$\begin{array}{llll}0 & \text { inlet conditions } & \mathrm{vr} & \text { vaporisation due to radiation } \\ \mathrm{d} & \text { drainage } & 1 & \text { liquid } \\ \mathrm{dr} & \text { radiation induced drainage } & \mathrm{cr} & \text { critical extension due to radiation }\end{array}$




\section{INTRODUCTION}

The spreading of foam on burning liquid surfaces is an important practical problem. A prediction of the spreading process would create a better basis for design of suppressing equipment and also for fire fighting tactics. It would also give information about the relative importance of different foam properties for a successful suppression. Very little has been done in formulating engineering models for the practical design and use of foam suppressing systems, though there is a substantial knowledge about foam flows in general, [1].

The problem with a spreading foam layer is analogous to the spreading of oil slicks on water. This latter problem has been treated in a number of papers, a review of the literature is given in [2].

The objective of the present study has been to develop a physical model describing the spreading process in order to enhance the understanding of the underlying phenomena. The model is based on the assumption that the spreading process is governed by a quasi steady balance between the driving force due to gravity and a resisting force due to viscous friction. Inertial effects are neglected. The mass transfer due to drainage and evaporation is decoupled from the spreading process by treating the foam flow as a pure volume flow with a constant mean density. The model is to be considered as a first order theory, future work may show it necessary to improve the model to account for certain effects not contained in the present formulation. Such effects could be inertia and changing shape of the foam layer. Furthermore, the model needs to be extended to include a calculation of the mass loss and the density once the spreading process has been solved. The bulk density can then be used to assess the capability of the foam to block fuel vapours to diffuse through the foam.

The present work is a first step in formulating a complete model describing foam spread on a burning surface which includes the prediction of the foam consumption due to radiation from the fire. The idea is to determine the foam properties and the friction factor in small scale experiments and then use the model to predict the full scale behaviour.

\section{THEORY}

The basic theory is outlined and applied to the case with a one dimensional foam flow (channel flow) without any radiative exposure. The foam is assumed to be supplied at $x=0$ with a constant volume flow rate $\dot{V}\left[\mathrm{~m}^{3} / \mathrm{m}, \mathrm{s}\right]$ (i.e. volume rate per unit width of a channel) and advancing in the positive $x$-direction, see Figure 1 . The instantaneous thickness of the spreading foam layer is denoted by $\boldsymbol{h}(\boldsymbol{x}, \boldsymbol{t})$, the thickness at $\boldsymbol{x}=0$ is $\boldsymbol{h}_{0}$, and the instantaneous location of the foam front is given by $x=L(t)$. In Figure 1 the submergence of the foam is indicated. In the following analysis this submergence is neglected and the interface between the foam and the liquid is assumed to be aligned with the undisturbed liquid surface. However, the effect of the submergence is accounted for in determining the hydrostatic driving force.

The viscosity of the foam is several orders of magnitude larger than the viscosity of water and ordinary hydrocarbon liquid fuels, [3]. This implies that the velocity gradients in the foam will be small compared to the gradients in the fluid and consequently the velocity in the foam layer can to a good approximation be assumed to be constant across the layer. The local velocity of the foam layer is denoted by $\boldsymbol{u}(\boldsymbol{x}, \boldsymbol{t})$.

Considering a unit width of the layer, the continuity equation for the foam flow can be expressed 
$\frac{\partial}{\partial t}(\rho h)+\frac{\partial}{\partial x}(\rho u h)=-G(x, t)$

In eq.(1) $\rho$ denotes the local density of the foam layer i.e. $\rho$ is generally a function of $\boldsymbol{x}$ and $t$. The function $G(x, t)$ denotes the mass loss due to drainage and evaporation and can be written

$G(x, t)=\dot{m}_{d}+\dot{m}_{d r}+\dot{m}_{v r}$

where $\dot{m}_{d}$ is the mass loss due to drainage, $\dot{m}_{d r}$ the additional drainage caused by radiation and $\dot{m}_{y r}$ the mass loss due to vaporisation.

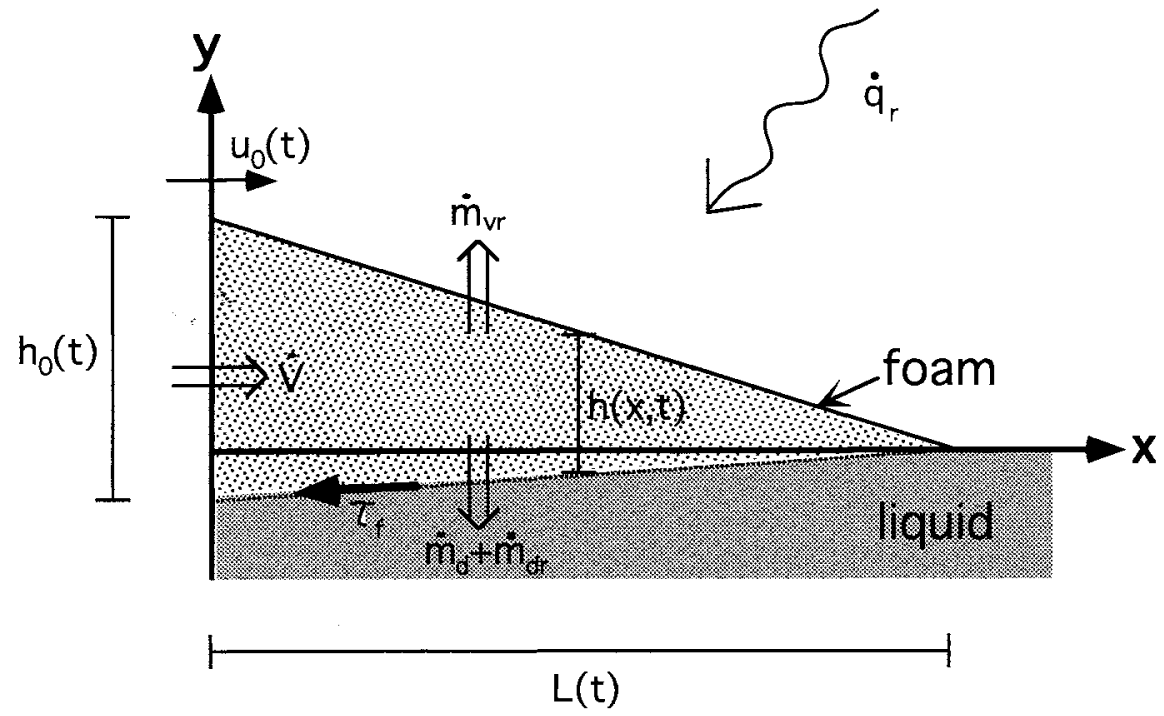

FIGURE 1 Schematic picture of a foam layer spreading on a liquid surface. The foam is applied from the left with a constant volume flow rate $\dot{V}$ and the spread is opposed by a friction force $\tau_{f}$. The foam is exposed to a radiative flux $\dot{\boldsymbol{q}}_{\boldsymbol{r}}$ causing an evaporation $\dot{m}_{y r}$ and a radiation induced drainage $\dot{m}_{d r}$ in addition to the conventional drainage $\dot{m}_{d}$.

Experimental results reported in [4] indicate that the drainage is an internal process in the foam that does not influence the layer thickness but merely the density. Furthermore, the experiments show that when a foam is exposed to radiation, part of the water in the foam will evaporate while part of the mass loss will appear as an increased drainage. The mass loss caused by the radiation is accompanied by a decrease in the layer thickness. The rate of decrease of the foam layer is uniquely determined by the radiative flux $\dot{\boldsymbol{q}}_{\boldsymbol{r}}$ and can be considered as being independent of the mass loss. These findings indicate the possibility of decoupling the variation of the foam thickness from the mass transport. 
The variation of the bulk density of the foam with account taken of the mass loss can be expressed

$h \frac{D \rho}{D t}=h\left(\frac{\partial \rho}{\partial t}+\boldsymbol{u} \frac{\partial \rho}{\partial x}\right)=-G(x, t)$

Carrying out the differentiation in eq.(1) and making use of (3) yield the following simplified continuity equation

$\frac{\partial \boldsymbol{h}}{\partial t}+\frac{\partial}{\partial \boldsymbol{x}}(\boldsymbol{u h})=0$

A balance equation for the axial momentum in this case takes the form

$\frac{\partial}{\partial t}(\rho \boldsymbol{u} h)+\frac{\partial}{\partial x}\left(\rho \boldsymbol{u}^{2} \boldsymbol{h}\right)=-\rho g\left(1-\frac{1}{S}\right) \boldsymbol{h} \frac{\partial \boldsymbol{h}}{\partial \boldsymbol{x}}-\tau_{f}$

Here the left hand side expresses the rate of change of inertia, the first term on the right hand side is the driving force caused by the hydrostatic pressure across the foam layer and the last term, $\tau_{f}$, denotes the resulting friction force. The latter term is a complicated function of several parameters describing the friction forces between the foam layer and the liquid underneath. The parameter $S$ denotes the ratio between the density of the water $\rho_{l}$ and the density of the foam $\rho$. This parameter is called the expansion ratio or foam number and is sometimes used as a quality parameter of the foam. In this analysis the foam number is treated as a constant.

In the momentum balance equation the bulk density serves as the link between the spreading process and the mass transport. To maintain the decoupling approximation the density is considered as constant and is introduced as a suitable mean value. Note the relationship between the foam density $\rho$ and the foam number $S$.

The foam spreading process is assumed to be quasi steady, determined by a balance between the friction and the driving forces. The momentum balance equation (5) then simplifies to

$$
\tau_{f}=-\rho g\left(1-\frac{1}{S}\right) h \frac{\partial h}{\partial x}
$$

The quasi steady assumption is partially vindicated by applying an order of magnitude analysis of the experimental results given in [5]. The analysis shows that the inertia terms in eq.(5) can be neglected compared to the terms on the right hand side provided the volume flow is sufficiently low or the spreading time is sufficiently long. No attempt has as yet been made to establish the exact limits when the approximation is valid.

The basic equations governing the foam spread are now given by eqs. (4) and (6). An additional condition is needed at the interface between the foam and the liquid to determine the viscous force $\tau_{f}$. A picture of the velocity fields set up in the foam and the liquid is shown in Figure 2. 
The retarding friction force can in the general case be written

$$
\tau_{f}=\mu_{l} \frac{\partial u_{l}}{\partial y}=\mu_{l} \cdot \frac{u}{\delta_{l}}
$$

where the gradient has been rewritten by introducing a fictitious boundary layer thickness $\delta_{l}$, $\boldsymbol{u}$ is the velocity at the interface i.e. the local foam speed. Suffix "l" denotes liquid, $\mu_{l}$ is the dynamic viscosity of the liquid and the co-ordinate $y$ is perpendicular to the plane of the spreading foam.

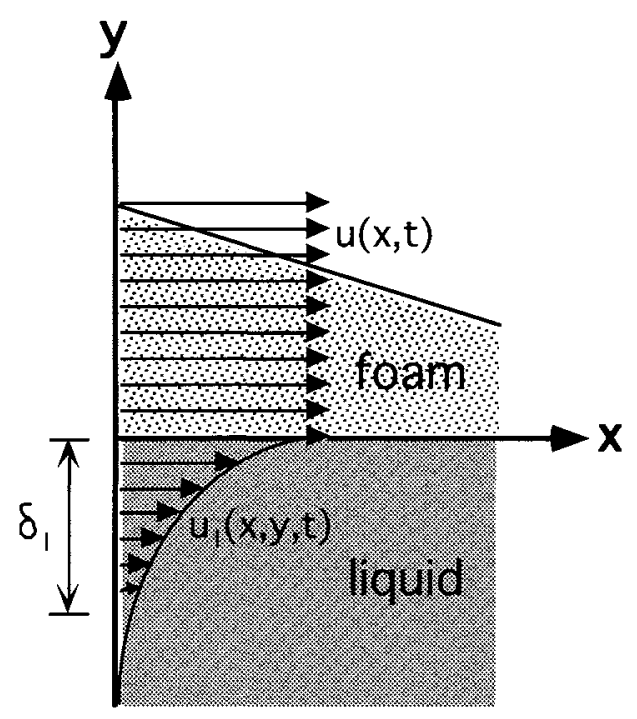

FIGURE 2 Velocity distributions in the foam and the liquid.

The boundary layer thickness can be estimated by making use of handbook formulas for the viscous friction in liquid flows, expressing the friction as a function of the local Reynolds number, see e.g. [6]. However, in view of all uncertainties appearing in the present model, this Reynolds number dependence is disregarded and the variation of $\delta_{l}$ is lumped into a friction constant $k_{f}$. The friction term can then simply be expressed

$$
\tau_{f}=k_{f} u
$$

Equating (6) and (8) yields

$$
\boldsymbol{u}=-\beta \boldsymbol{h} \frac{\partial \boldsymbol{h}}{\partial \boldsymbol{x}}
$$

where the friction parameter $\beta$ is defined as 
$\beta=\frac{\rho g\left(1-\frac{1}{S}\right)}{k_{f}}$

Another boundary condition to the present problem is provided by the foam flow at the inlet. Assuming a constant volume flow $\dot{\boldsymbol{V}}$, the boundary condition at $\boldsymbol{x}=0$ can be expressed

$\boldsymbol{u}_{0} \boldsymbol{h}_{0}=\dot{\boldsymbol{V}}$

where suffix " 0 " indicates inlet conditions.

\section{SOLUTION PROCEDURE}

An approximate solution will be formulated based on the integral solution technique [6]. In this technique information about the functional form of the solution is needed e.g. the shape of the foam layer. From [5] it is evident that the foam layer to a good approximation can be assumed to have a triangular shape during the spreading history. Thus, the thickness distribution of the foam layer is assumed to be

$h(x, t)=h_{0}(t)\left(1-\frac{x}{L(t)}\right)$

where account has been taken of the fact that the foam layer has zero thickness at the front.

Here $\boldsymbol{h}_{0}$ is the thickness at $\boldsymbol{x}=0$ and $\boldsymbol{L}(\boldsymbol{t})$ is the instantaneous location of the foam front.

With this assumed foam distribution it is possible to formulate an integral balance equation by integrating equation (4) along the foam layer from $x=0$ to $x=L$.

The resulting equation takes the form

$\frac{d}{d t}\left(\int_{0}^{L} h \cdot d x\right)=-\int_{0}^{L} \frac{\partial}{\partial x}(u h) \cdot d x=u_{0} h_{0}$

Inserting (12) into (13) yields after integration (noting that $\boldsymbol{u}_{0} \boldsymbol{h}_{0}=\dot{\boldsymbol{V}}$ is constant)

$$
h_{0} L=2 \dot{V} \cdot t
$$

Another relation between $\boldsymbol{u}_{0}, \boldsymbol{h}_{0}$, and $\boldsymbol{L}$ can be obtained by making use of (9) and performing a formal mean value formation along the foam layer thus giving

$$
u_{0}=\bar{\beta} \cdot \frac{h_{0}^{2}}{L}
$$


Note that the friction parameter $\bar{\beta}$ in (15) is different from the one in (9) due to the mean value formation. This is only a formality and the bar will be omitted in the following.

Now we have three equations at our disposal, (11), (14) and (15) to determine the three unknowns $u_{0}, h_{0}$, and $L$.

\section{ONE DIMENSIONAL FOAM FLOW WITHOUT RADIATION}

In this case equations (11), (14) and (15) can be directly used to formulate the solutions which take the form

$$
\begin{aligned}
& \boldsymbol{h}_{0}=\left(\frac{4 \dot{\boldsymbol{V}}^{2}}{\beta}\right)^{1 / 4} \cdot \boldsymbol{t}^{1 / 4} \\
& \boldsymbol{L}=\left(4 \beta \dot{\boldsymbol{V}}^{2}\right)^{1 / 4} \cdot \boldsymbol{t}^{3 / 4}
\end{aligned}
$$

No direct comparison with experimental results are given for this case because appropriate experiments are missing.

\section{AXISYMMETRIC FOAM SPREAD WITHOUT RADIATION}

The basic equations in this case take the form

$$
\begin{aligned}
& \frac{\partial}{\partial t}(\rho r h)+\frac{\partial}{\partial r}(\rho r u h)=-r G(r, t) \\
& \frac{\partial}{\partial t}(\rho u h)+\frac{1}{r} \cdot \frac{\partial}{\partial r}\left(\rho r u^{2} h\right)=-\rho g\left(1-\frac{1}{S}\right) h \frac{\partial h}{\partial r}-\tau_{f}
\end{aligned}
$$

where $\boldsymbol{r}$ now denotes the radial co-ordinate.

These equations can be simplified in exactly the same way as for the one dimensional case thus giving

$$
\frac{\partial}{\partial t}(\boldsymbol{r} h)+\frac{\partial}{\partial \boldsymbol{r}}(\boldsymbol{r u h})=0
$$

for the continuity equation.

The momentum equation turns out to be exactly the same as before i.e. given by (6) with $x$ replaced by $r$.

In this case it is assumed that the foam is introduced in a circular cylinder with radius $\boldsymbol{R}_{0}$ 
and with a constant volume flow $\dot{V}\left[\mathrm{~m}^{3} / \mathrm{s}\right]$ (note the different definition of $\dot{V}$ compared to the one dimensional case). The boundary condition for $\boldsymbol{r}=\boldsymbol{R}_{0}$ then reads

$$
2 \pi R_{0} u_{0} h_{0}=\dot{V}
$$

Integration of (20) between $\boldsymbol{r}=\boldsymbol{R}_{0}$ and the outer edge of the foam layer at $\boldsymbol{r}=\boldsymbol{R}$ gives

$$
\frac{d}{d t}\left(\int_{R_{0}}^{R} r h \cdot d r\right)=-\int_{R_{0}}^{R} \frac{\partial}{\partial r}(r u h) \cdot d r=R_{0} u_{0} h_{0}
$$

The shape of the foam layer is again assumed to be linear i.e.

$$
\boldsymbol{h}(\boldsymbol{r}, \boldsymbol{t})=\boldsymbol{h}_{0}(\boldsymbol{t})\left(1-\frac{\boldsymbol{r}}{\boldsymbol{R}(\boldsymbol{t})}\right)
$$

Introducing this into (22) and making use of (21) gives after integration

$$
h_{0} R^{2}=\frac{3 \dot{V} \cdot t}{\pi}
$$

In the derivation of (24) it has also been assumed that $\boldsymbol{R}_{0}<<\boldsymbol{R}$ which is an acceptable approximation except for the very first part of the spreading process.

Applying the same frictional relationship as in (9) and performing the mean value formation leads to

$$
\boldsymbol{u}_{0}=\beta \cdot \frac{\boldsymbol{h}_{0}{ }^{2}}{\boldsymbol{R}}
$$

The resulting solutions are obtained by combining (21), (24) and (25) i.e.

$$
\begin{aligned}
& \boldsymbol{h}_{0}=\left(\frac{27 \dot{\boldsymbol{V}}^{3}}{4 \pi^{3} \beta^{2} \boldsymbol{R}_{0}{ }^{2}}\right)^{1 / 7} \cdot t^{1 / 7} \\
& \boldsymbol{R}=\left(\frac{18}{\pi^{2}} \cdot \beta \boldsymbol{R}_{0} \dot{\boldsymbol{V}}^{2}\right)^{1 / 7} \cdot \boldsymbol{t}^{3 / 7}
\end{aligned}
$$

\section{COMPARISON WITH EXPERIMENTS}

The comparison between theory and experiments is based on the axisymmetric case to avoid any disturbances of wall effects. Experiments have been conducted for two different types of foam, Detergent and AFFF, in a circular water basin with a diameter of $20 \mathrm{~m}$, [5]. Figures 3 7 show a comparison between the measured and the calculated foam thickness and extension according to equations (26) - (27). The friction coefficient $\boldsymbol{k}_{f}=0.1\left[\mathrm{~N} / \mathrm{m}^{3}\right]$ has been 
determined by making a best fit between theory and experiments for the set of data used in Figures $3-7$. The foam expansion number $S$ and the corresponding foam density used are as measured in the experiments.

A theoretical estimate of the friction coefficient can be obtained by using the theory for flat plate boundary layers, [6]. With Reynolds numbers based on the foam extension as the characteristic length, the rate of growth from Figure $3-7$ as the characteristic velocity and with table values for the viscosity of water, one gets $\boldsymbol{k}_{\boldsymbol{f}}$ values ranging from 0.05 to 0.15 depending on the time. Thus the selected value 0.1 is a reasonable mean value over the time span considered.

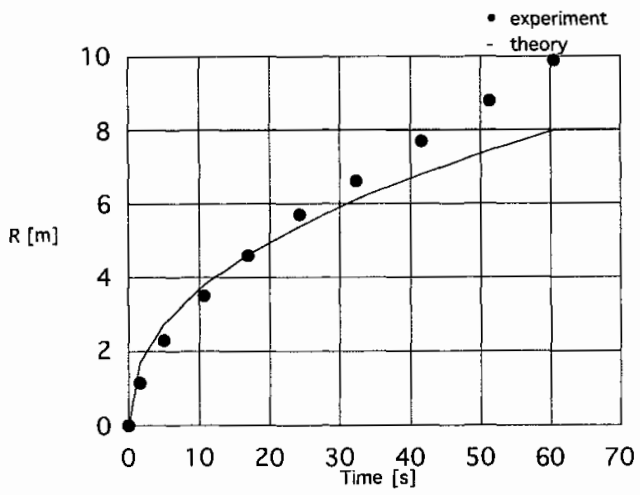

FIGURE 3 Comparison between predicted and measured extension for Detergent foam. Volume flow $25 \mathrm{l} / \mathrm{s}, \boldsymbol{S}=7.5$ and $\boldsymbol{k}_{\boldsymbol{f}}=0.1\left[\mathrm{~N} / \mathrm{m}^{3}\right]$.

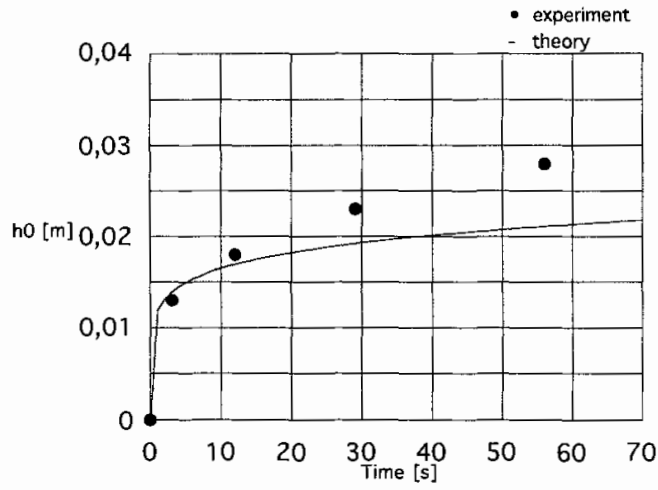

FIGURE 4 Comparison between predicted and measured layer thickness for Detergent foam. Volume flow $25 \mathrm{l} / \mathrm{s}, S=7.5$ and $\boldsymbol{k}_{f}=0.1\left[\mathrm{~N} / \mathrm{m}^{3}\right]$. 


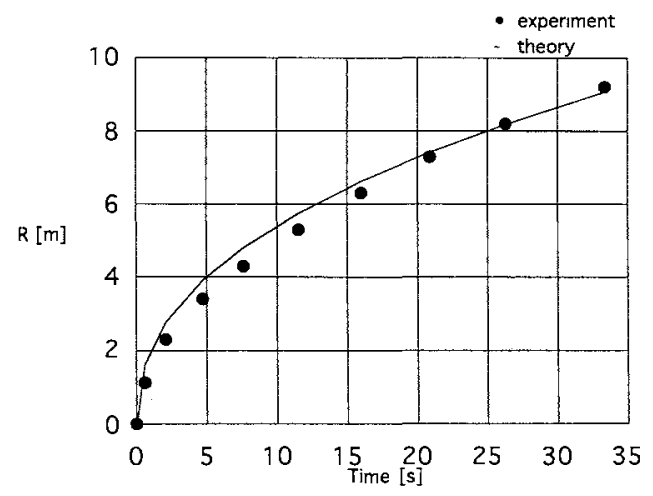

FIGURE 5 Comparison between predicted and measured extension for Detergent foam. Volume flow $107 \mathrm{l} / \mathrm{s}, \boldsymbol{S}=12.3$ and $\boldsymbol{k}_{f}=0.1\left[\mathrm{~N} / \mathrm{m}^{3}\right]$.

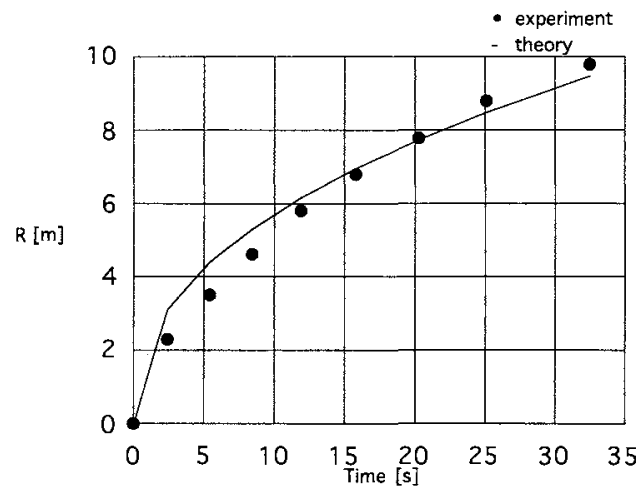

FIGURE 6 Comparison between predicted and measured extension for AFFF foam. Volume flow $194 \mathrm{l} / \mathrm{s}, \boldsymbol{S}=28.9$ and $\boldsymbol{k}_{f}=0.1\left[\mathrm{~N} / \mathrm{m}^{3}\right]$. 


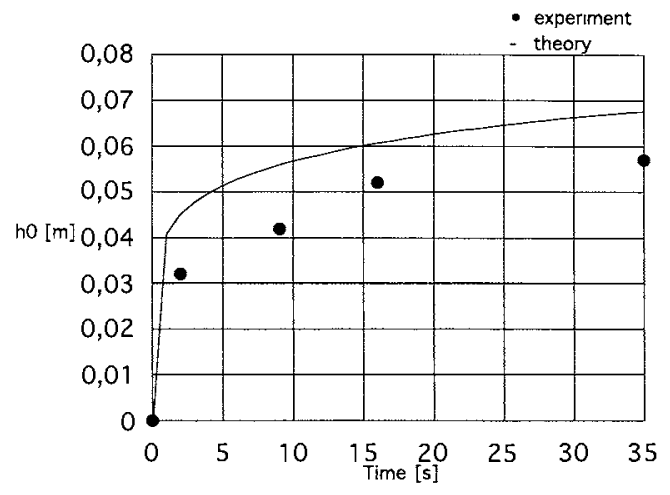

FIGURE 7 Comparison between predicted and measured layer thickness for AFFF foam. Volume flow $194 \mathrm{l} / \mathrm{s}, \boldsymbol{S}=28.9$ and $\boldsymbol{k}_{f}=0.1\left[\mathrm{~N} / \mathrm{m}^{3}\right]$.

As is seen from Figures $3-7$ the agreement is acceptable over a wide range of volume flow rates and foam expansion numbers. The model tends to under predict the spreading rate which is probably a result of the neglected initial momentum of the foam. The prediction of the layer thickness is less god than the prediction of the extension. This is due to the assumed linear thickness distribution of the foam layer. However, overall the model predicts the spreading process reasonably well and indicates the possibility of using a constant lumped friction coefficient for describing the resistance against the spreading foam.

\section{CONCLUDING REMARKS}

An approximate model describing the spreading of foams on liquid surfaces has been formulated. The model is based on simplifying assumptions regarding the influence of inertia and viscous friction. Nevertheless the model seems to contain the main ingredients necessary for predicting the spreading process once the friction constant has been determined. Preliminary comparisons with experiments are promising. Thus, it seems feasible to formulate a simple theoretical model for foam spreading along the lines presented here, at least with sufficient accuracy for engineering purposes.

\section{Acknowledgements}

Financial and technical support by the Swedish National Rescue Services Board is gratefully acknowledged. 


\section{REFERENCES}

1. Kraynik A. M., "Foam Flows", Annual Review of Fluid Mechanics, 20, 325-357, 1988.

2. Hoult D. P., "Oil Spreading on the Sea", Annual Review of Fluid Mechanics, 4 , 341$368,1972$.

3. Kroezen A. B. J., Groot Wassink J. and Schipper C. A. C., "The flow properties of foam", Journal of the Society of Dyers and Colourists, 104, 393-396, 1988.

4. Persson H., "Fire Extinguishing Foams - Resistance Against Heat Radiation", SP Report 1993:54, 1993.

5. Dahlberg M., "Foam Spread Experiments on a Liquid Surface", SP Report 1994:16, 1994

6. Eckert E.R.G. and Drake Jr R.M., Heat and Mass Transfer, McGraw-Hill Co, New York, 1959. 